\title{
Prevalence and factors associated with postpartum depression at a primary healthcare facility in Eswatini
}

\begin{tabular}{|c|c|}
\hline \multicolumn{2}{|c|}{ 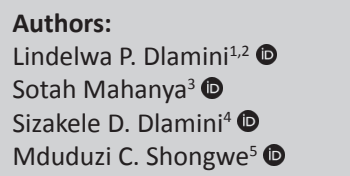 } \\
\hline \multicolumn{2}{|c|}{ 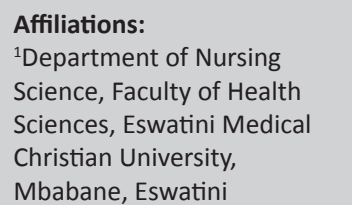 } \\
\hline $\begin{array}{l}{ }^{2} \text { International } \\
\text { Program in Nu } \\
\text { Department of } \\
\text { National Chen } \\
\text { University, Tair }\end{array}$ & $\begin{array}{l}\text { Advanced } \\
\text { rsing, } \\
\text { f Nursing, } \\
\text { g Kung } \\
\text { nan, Taiwan }\end{array}$ \\
\hline \multicolumn{2}{|c|}{$\begin{array}{l}{ }^{3} \text { Good Shepherd College of } \\
\text { Nursing, Siteki, Eswatini }\end{array}$} \\
\hline \multicolumn{2}{|c|}{$\begin{array}{l}{ }^{4} \text { Department of Psychology, } \\
\text { Faculty of Applied Social } \\
\text { Sciences, Eswatini Medical } \\
\text { Christian University, } \\
\text { Mbabane, Eswatini }\end{array}$} \\
\hline \multicolumn{2}{|c|}{$\begin{array}{l}{ }^{5} \text { Department of Midwifery } \\
\text { Science, Faculty of Health } \\
\text { Sciences, University of } \\
\text { Eswatini, Mbabane, Eswatini }\end{array}$} \\
\hline \multicolumn{2}{|c|}{$\begin{array}{l}\text { Corresponding author: } \\
\text { Mduduzi Shongwe, } \\
\text { mduyaye@gmail.com }\end{array}$} \\
\hline $\begin{array}{l}\text { Dates: } \\
\text { Received: } 24 \mathrm{~A} \\
\text { Accepted: } 21 \mathrm{~A} \\
\text { Published: } 24\end{array}$ & $\begin{array}{l}\text { pr. } 2019 \\
\text { Aug. } 2019 \\
\text { Oct. } 2019\end{array}$ \\
\hline \multicolumn{2}{|c|}{$\begin{array}{l}\text { How to cite this article: } \\
\text { Dlamini LP, Mahanya S, } \\
\text { Dlamini SD, Shongwe MC. } \\
\text { Prevalence and factors } \\
\text { associated with postpartum } \\
\text { depression at a primary } \\
\text { healthcare facility in Eswatini. } \\
\text { S Afr J Psychiat. 2019;25(0), } \\
\text { a1404. https://doi.org/ } \\
\text { 10.4102/sajpsychiatry. } \\
\text { v25i0.1404 }\end{array}$} \\
\hline \multicolumn{2}{|l|}{ Read online: } \\
\hline 品战品 & $\begin{array}{l}\text { Scan this QR } \\
\text { code with your } \\
\text { smart phone or } \\
\text { mobile device } \\
\text { to read online. }\end{array}$ \\
\hline
\end{tabular}

Background: Routine mental health screening has not been integrated into maternal and child health $(\mathrm{MCH})$ services in many developing countries, including in Eswatini (formerly Swaziland). As a result, the burden of postpartum depression (PPD) is not well understood and thus PPD remains untreated in such settings.

Aim: To describe the prevalence and factors associated with PPD among women seeking postnatal and child welfare services at a primary healthcare facility in Eswatini.

Setting: The study was conducted at the King Sobhuza II Public Health Unit in Manzini, Eswatini.

Methods: This was a cross-sectional study that used convenience sampling and the Edinburgh Postnatal Depression Scale (EPDS) to screen for depression among 114 mothers during the first 6 weeks of postpartum at the King Sobhuza II Public Health Unit, Manzini, Eswatini. Multiple logistic regression analysis was conducted to determine sociodemographic and clinical factors associated with PPD.

Results: A majority of the participants were older than 24 years (52.6\%) and unemployed (64.9\%), whereas $47.4 \%$ screened positive for PPD ( $\geq 13$ score). Adjusting for other covariates, those who were unemployed (odds ratio $[\mathrm{OR}]=3.20,95 \%$ confidence interval $[\mathrm{CI}] 1.17-8.79$ ) and with poor social support from their partners ( $\mathrm{OR}=9.41,95 \% \mathrm{CI}$ : 3.52-25.14) were more likely to be depressed, while those who attended antenatal classes fewer than four times were less likely to be depressed (OR $=0.32,95 \%$ CI $0.11-0.92)$.

Conclusion: We found a high prevalence of PPD. There is a need to introduce routine maternal mental health screening during the postpartum period to ensure early detection and treatment of PPD.

Keywords: maternal mental health; perinatal depression; postnatal depression; postpartum; postpartum depression.

\section{Introduction}

Postpartum depression (PPD) is one of the most common complications of childbearing, occurring in $13 \%$ of postpartum women worldwide. ${ }^{1}$ This is even higher in developing countries as $15.6 \%$ and $19.8 \%$ of women experience mental health disorders during pregnancy and after childbirth, respectively. ${ }^{2}$ Postpartum depression is defined as a major depressive episode with peripartum onset (i.e. the most recent episode occurring during pregnancy as well as in the 4 weeks following delivery), accompanied by a depressed or sad mood, marked loss of interest in virtually all activities, significant weight loss or gain, insomnia or hypersomnia, psychomotor agitation or retardation, fatigue or loss of energy, feelings of worthlessness or guilt, diminished ability to think or concentrate, and recurrent thoughts of death. ${ }^{3}$ In this study, we limited the onset specifier to 6 weeks after delivery because our interest was in quantifying the prevalence of the major depressive disorder during the postpartum period, which is 6 weeks. In subSaharan Africa, studies have reported varying prevalence of PPD: 6.6\% in Uganda, 50.8\% in the Democratic Republic of Congo ${ }^{4}$ and $33.3 \%$ in Nigeria. ${ }^{5}$ In KwaZulu-Natal, South Africa, a setting closer to Eswatini (formerly Swaziland), about $35.0 \%$ experience depression postpartum. ${ }^{6}$ These figures show that maternal mental health disorders are common in this region, disputing assumptions in 20th century literature, ${ }^{7}$ which postulated that somehow women in Africa were protected from perinatal mental disorders due to traditional rituals and other cultural factors. 
Research $^{8}$ has shown that PPD victims are twice as likely to experience future episodes of depression over a 5-year period. A recent systematic review ${ }^{9}$ revealed that the risk factors for PPD include socio-economic disadvantage, unintended pregnancy, being younger, being unmarried, lacking intimate partner empathy and support, having hostile in-laws, experiencing intimate partner violence, having insufficient emotional and practical support and, in some settings, giving birth to a female baby, and having a history of mental health problems.

Findings on the mode of delivery and PPD are mixed: one study ${ }^{10}$ found that vaginal delivery was a risk factor for PPD, others ${ }^{11,12}$ found no direct link and yet another study ${ }^{13}$ found that women who delivered by caesarian section were at an increased risk of PPD. In Africa, the unique risk factors for PPD include poor infant nutritional status, low infant birth weight, shorter duration of breastfeeding, diarrhoeal diseases, poor self-rated health, respiratory illness, home delivery, reduced quality of interaction between mothers and infants, ${ }^{14}$ and poor linkage to HIV care. ${ }^{15}$ However, attaining a high education level, having a permanent job and having a kind and trustworthy intimate partner are assumed to be protective against PPD. ${ }^{9}$

In Eswatini, mental health issues have not been in the forefront in terms of advocacy and prioritisation in the Ministry of Health (MoH)'s programming agenda, which has resulted in limited mental health service delivery to the general population. For example, the country has only one psychiatric hospital and one psychiatrist serving the whole population of about 1.1 million. Currently, there is no national mental health policy, and mental health screening during pregnancy and postpartum have not been integrated in the delivery of maternal and child health $(\mathrm{MCH})$ services. Identifying and treating PPD is necessary because if severe, it places the mother at an increased risk for suicidal and infanticidal behaviour. ${ }^{16}$ Additionally, affected mothers cannot function properly and, as a result, their children's growth and development may be negatively affected. ${ }^{2}$

Findings from this study will help provide baseline data for the $\mathrm{MoH}$ and the Sexual Reproductive Health (SRH) programme in Eswatini on the burden and correlates of PPD in the country. Understanding the factors associated with PPD will provide healthcare workers and programmers with indicators to include when developing maternal mental health screening tools. When clinicians are informed about the risk factors for PPD, they will be in a better position to tailor maternal mental health interventions for mothers based on those identified factors. Currently, there is limited published research from Eswatini on mental health in general, let alone on maternal mental health, which has contributed to the lack of mental health and maternal mental health policy and programme development. The only published evidence about maternal depression from Eswatini is on antepartum depression (APD) ${ }_{1}^{15}$ which was found to be at $22.7 \%$. However, it is unclear whether the magnitude of the depression persists postpartum or not. As a result, we conducted this study to describe the burden of PPD and its associated factors among postpartum women seeking postnatal and child welfare services at the King Sobhuza II Public Health Unit (KS II) in Eswatini.

\section{Methods Study design and setting}

A cross-sectional study design was employed within the quantitative research approach. The study was conducted at the KS II PHU. The country's healthcare delivery system is based on the primary healthcare strategy and is loosely organised in a four-tier system. The KS II PHU is a level II facility located in the central part of Eswatini, in Manzini. This facility provides a range of services, such as antenatal care, postnatal care, family planning, child welfare, outpatient curative care, and so forth. The KS II PHU was purposively selected because among all the level II healthcare facilities in this region, it has one of the highest number of patient visits per year in this region. ${ }^{17}$ The Manzini region was chosen because it has the highest number of the country's population and resembles almost all the characteristics of the other regions, as it is host to the major industrial hub as well as the biggest city (Manzini Municipality), yet it still has remote rural areas within its boundaries. ${ }^{18}$ The KS II PHU is located in the busiest and urban part of the city and services residents from urban, peri-urban as well as from rural surroundings of the city.

\section{Study population and sample size}

The study population included mothers who had come to seek postnatal or child welfare services at this facility who were between 7 days and 6 weeks of the postpartum period in the months of June-July 2017. In order to be part of this study, the women had to be Swazi, be 18 years old or older and should have given birth not less than 7 days before the time of data collection, but within 6 weeks since giving birth. Women who had given birth less than 7 days before the time of data collection were excluded from the study to avoid information bias through misclassification as it would be difficult for women to distinguish postpartum blues (which usually occur in the first 3-5 days after childbirth) from depressive symptoms.

The sample size for this study was estimated using Raosoft ${ }^{\circledR}$ online sample size calculator ${ }^{19}$ assuming the following parameters: a margin of error of 5\%, confidence level of $95 \%$, population size of 12500 deliveries in the year 2015 in the Manzini region ${ }^{17}$ and an estimated response distribution of $22.7 \%$ (based on the prevalence of antepartum depression in Eswatini); ${ }^{15}$ the minimum desired sample size for this study was 264. However, 118 participants were approached to participate in the study, two refused and another two did not return to the private room after receiving their clinical services, making the eventual sample size for this study 114, indicating a response rate of about $97 \%$. 


\section{Recruitment and data collection procedures}

We employed convenience sampling to recruit the women and data were collected by the first author through face-toface interviews using a well-structured questionnaire. After permission was granted by the nurse manager of the facility, the senior nurse introduced the first author to all the mothers in the waiting area during the health education session that is usually held before the start of work on a daily basis in this facility.

Participants were identified (i.e. those carrying babies) while queuing on the bench leading to the clinic rooms and were approached to participate in the study. If the participant expressed willingness to participate, they were invited to a private room that was assigned by the facility management to conduct the interviews.

Participants were interviewed after they had already received the care they had come for at the clinic in order not to disturb the nurses' work routine. The interviews were conducted in SiSwati and each interview took an average of $20 \mathrm{~min}$ including $5 \mathrm{~min}$ for obtaining written informed consent prior to each interview.

\section{Measures}

A well-structured, researcher-administered questionnaire was utilised to collect the data. The questionnaire had two sections: Section A asked participants to report their sociodemographic characteristics as well as other social and clinical factors related to PPD identified during literature review and Section B consisted of the Edinburgh Postnatal Depression Scale (EPDS) with 10 items screening for PPD, with the last one also screening for suicidal ideation. ${ }^{20}$ Participants responded to a 4-point Likert scale (0-3). The total score was calculated by summing up all item scores with the total possible score ranging from 0 to 30 . Women with an EPDS score of 13 or more were classified as having depressive symptoms. ${ }^{20}$ Prior to this study, the EPDS had not been tested among postpartum women in Eswatini; however, it has been widely used and validated in diverse African settings including among antepartum women in Eswatini. ${ }^{15}$. A systematic review and meta-analysis of 10 African studies ${ }^{21}$ estimated the median coefficient alpha of the EPDS to be 0.84 (interquartile range $0.71-0.87$ ), whereas at a cut-off score of $\geq 9$ among 14 African studies, it demonstrated a pooled sensitivity of 0.94 (95\% confidence interval [CI]: 0.68-0.99) and a pooled specificity of 0.77 (95\% CI 0.59-0.88) with higher cut-off scores yielding greater specificity against a lower sensitivity. ${ }^{21}$ In this study, the EPDS reached an acceptable reliability with a Cronbach's $\alpha$ of 0.81 .

\section{Data analysis}

Data were analysed using IBM Statistical Package for Social Sciences (SPSS) for Windows, version $20 .{ }^{22}$ For bivariate analysis, we performed Pearson's chi-square tests to examine significant sociodemographic differences by PPD status, whereas multiple logistic regression analysis was performed using the forced entry method ${ }^{23}$ to build the final model. We computed adjusted odds ratios (AORs) and 95\% CIs at an alpha level of 0.05 . Selection of variables to be included in the final model was guided by the known clinical importance of the variables (based on literature), their contribution score in the model as well as an alpha level of $<0.25$ in bivariate analyses. ${ }^{24}$ Model diagnostics were assessed using: (1) Hosmer and Lemeshow test, $\chi^{2}$ (degree of freedom, $d f=8)=7.25, p=0.51$, which is $>0.05$ indicating that the model fitted the data well, (2) the pseudo- $R^{2}$, (3) the $p$-value of the model $\chi^{2}$ (both shown in Table 2) and (3) Cook's deviance values (all $<1$ ) and standardised residuals (all within \pm 2.58 ). We assessed for multicollinearity between the covariates by fitting the same model using the linear regression procedure ${ }^{23}$ to obtain tolerance (all $<1$ ) and variance inflation factor $(\mathrm{VIF})$ values $($ all $<10)$ indicating the absence of multicollinearity in the final model.

\section{Ethical considerations}

Ethical clearance was obtained from the National Health Research Review Board (NHRRB) in Eswatini (Ref: MH/599C/ IRB0009688/NHRRB 548/17). Participation in the study was voluntary and participants were assured that they could withdraw from the study at any time. Considering the potential distressing effects of some of the questions in the EPDS, the first author (a nurse by training) debriefed all the participants after completing the interviews by offering information about where they could seek psychological help if they felt that the questionnaire had evoked some emotional distress. There were two such cases in this study, and together with 20 others who expressed suicidal ideations, they were encouraged to seek further psychological management at the National Psychiatric Referral Hospital (NPRH), which is located about $1 \mathrm{~km}$ from the study site. Consent was obtained from the mothers to link them with nurses at the KS II PHU who in turn referred them to the NPRH as per local referral clinical procedures. All other participants who screened positive to the EPDS were also encouraged to go for a psychological consult at the NPRH at any day following the interview.

\section{Results \\ Participants' characteristics}

A total of 114 postpartum women participated in this study. A majority of them were older than 24 years $(n=60,52.6 \%)$, were unemployed $(n=74,64.9 \%)$, were multipara $(n=71$, $62.3 \%)$, had not planned the pregnancy for the current child ( $n=79,69.3 \%)$, had no history of gender-based violence $(n=91,79.8 \%)$ and reported receiving good social support from their partners $(n=68,59.6 \%)$. In bivariate analyses, Chi-square tests showed that depressed women were statistically significantly different from those who were not depressed by employment status $\left(\chi^{2}=3.78, d f=1, p=0.05\right)$, planned pregnancy status $\left(\chi^{2}=5.15, d f=1, p=0.02\right)$ and social support from the partner $\left(\chi^{2}=25.51, d f=1, p<0.001\right)$. There were no statistically significant differences between the two 
groups of women with respect to other sociodemographic and clinical factors in the other bivariate analyses (Table 1).

\section{Prevalence of postpartum depression}

As shown in Table 1, nearly half $(47.4 \%, n=54)$ of the participants screened positive for PPD (i.e. had a score of 13 or more on the EPDS). Worth noting is that about $22(19 \%)$ of the 114 participants also reported to have had thoughts of harming themselves (suicidal ideation) and/or the baby (infanticidal ideation) during the postpartum period (data not shown).

\section{Factors associated with postpartum depression}

We found that those who were unemployed (AOR $=3.20$, $95 \%$ CI 1.17-8.79, $p=0.03$ ) versus employed, and those who reported poor social support from their partners $(\mathrm{AOR}=9.41$, 95\% CI 3.52-25.14, $p<0.001)$ compared to those who had good social support, had higher odds of screening positive for PPD adjusting for other covariates in the model. To the contrary, those who attended antenatal classes fewer than four times were less likely to be depressed (AOR $=0.33,95 \%$ CI: $0.11-0.92, p=0.04)$ compared to those who attended antenatal classes four or more times adjusting for other covariates in the model. Other sociodemographic and clinical variables in the model did not show statistical significance (Table 2).

\section{Discussion}

This study found that the prevalence of PPD is high and that PPD is associated with employment status, antenatal care attendance and social support. Even though the prevalence

TABLE 1: Distribution of participants' characteristics by postpartum depression status $(N=114)$.

\begin{tabular}{|c|c|c|c|c|c|c|c|c|}
\hline \multirow[t]{2}{*}{ Characteristics } & \multicolumn{2}{|c|}{ Total } & \multicolumn{2}{|c|}{ Depressed $(n=54)$} & \multicolumn{2}{|c|}{ Not depressed $(n=60)$} & \multirow[t]{2}{*}{$\chi^{2}$} & \multirow[t]{2}{*}{$p$} \\
\hline & $n$ & $\%$ & $n$ & $\%$ & $n$ & $\%$ & & \\
\hline Maternal age (in years) & & & & & & & 0.29 & 0.59 \\
\hline $18-24$ & 54 & 47.4 & 27 & 50 & 27 & 45 & & \\
\hline $25-45$ & 60 & 52.6 & 27 & 50 & 33 & 55 & & \\
\hline Employment status & & & & & & & 3.78 & 0.05 \\
\hline Employed/self-employed & 40 & 35.1 & 14 & 25.9 & 26 & 43.3 & & \\
\hline Unemployed & 74 & 64.9 & 40 & 74.1 & 34 & 56.7 & & \\
\hline Parity & & & & & & & 0.02 & 0.89 \\
\hline Primipara & 43 & 37.7 & 20 & 37 & 23 & 38.3 & & \\
\hline Multipara & 71 & 62.3 & 34 & 63 & 37 & 61.7 & & \\
\hline Antenatal care attendance & & & & & & & 0.63 & 0.43 \\
\hline$<4$ times & 38 & 33.3 & 16 & 29.6 & 22 & 36.7 & & \\
\hline$\geq 4$ times & 76 & 66.7 & 38 & 70.4 & 38 & 63.3 & & \\
\hline Method of delivery & & & & & & & - & $0.31^{\mathrm{a}}$ \\
\hline Vaginal delivery & 104 & 91.2 & 51 & 94.4 & 53 & 88.3 & & \\
\hline Caesarean section & 10 & 8.8 & 3 & 5.6 & 7 & 11.7 & & \\
\hline Gestational age at delivery & & & & & & & 0.06 & 0.82 \\
\hline Term & 94 & 82.5 & 45 & 83.3 & 49 & 81.7 & & \\
\hline Pre-/post-term & 20 & 17.5 & 9 & 16.7 & 11 & 18.3 & & \\
\hline Had planned the pregnancy & & & & & & & 5.15 & 0.02 \\
\hline Yes & 35 & 30.7 & 11 & 20.4 & 24 & 40 & & \\
\hline No & 79 & 69.3 & 43 & 79.6 & 36 & 60 & & \\
\hline Maternal HIV status & & & & & & & 1.04 & 0.31 \\
\hline Positive & 43 & 37.7 & 23 & 42.6 & 20 & 33.3 & & \\
\hline Negative & 71 & 62.3 & 31 & 57.4 & 40 & 66.7 & & \\
\hline History of GBV & & & & & & & 3.68 & 0.06 \\
\hline Yes & 23 & 20.2 & 15 & 27.8 & 8 & 13.3 & & \\
\hline No & 91 & 79.8 & 39 & 72.2 & 52 & 86.7 & & \\
\hline Social support from partner & & & & & & & 25.51 & $<0.001$ \\
\hline Good & 68 & 59.6 & 19 & 35.2 & 49 & 81.7 & & \\
\hline Poor & 46 & 40.4 & 35 & 64.8 & 11 & 18.3 & & \\
\hline Sex of newborn & & & & & & & 2.25 & 0.13 \\
\hline Male & 57 & 50 & 23 & 42.6 & 34 & 56.7 & & \\
\hline Female & 57 & 50 & 31 & 57.4 & 26 & 43.3 & & \\
\hline Condition of baby at birth & & & & & & & - & $1.00 \dagger$ \\
\hline Normal/healthy baby & 107 & 93.9 & 51 & 94.4 & 56 & 93.3 & & \\
\hline Sick baby/congenital abnormality & 7 & 6.1 & 3 & 5.6 & 4 & 6.7 & & \\
\hline History of mental illness (excluding epilepsy) & & & & & & & NC & NC \\
\hline Yes & 6 & 5.3 & 4 & 7.4 & 2 & 3.3 & & \\
\hline No & 106 & 93 & 49 & 90.7 & 57 & 95 & & \\
\hline Do not know & 2 & 1.8 & 1 & 1.9 & 1 & 1.7 & & \\
\hline
\end{tabular}

GBV, gender-based violence; HIV, human immunodeficiency virus; NC, not computed due to few observations. $\dagger$, Fisher's exact test. 
TABLE 2: Multiple logistic regression model for factors associated with postpartum depression $(N=114)$.

\begin{tabular}{lccccc}
\hline Variable & $\boldsymbol{\beta}$ & SE & AOR & $95 \% \mathrm{Cl}$ & $p$ - \\
\hline Employment status & & & & & \\
Employed & - & - & Ref & - & - \\
Unemployed & $1.12^{*}$ & 0.52 & 3.20 & $1.17-8.79$ & 0.02 \\
Pregnancy planned & & & & & \\
Yes & - & - & Ref & - & - \\
No & 0.66 & 0.51 & 1.96 & $0.72-5.33$ & 0.19 \\
Social support from partner & & & & & \\
Good & - & - & Ref & - & - \\
Poor & $2.24 *$ & 0.50 & 9.41 & $3.52-25.14$ & $<0.001$ \\
History of GBV & & & & & \\
Yes & 0.89 & 0.59 & 2.44 & $0.76-7.81$ & 0.13 \\
No & - & - & Ref & - & - \\
Antenatal care attendance & & & & & \\
$<4$ times & $-1.13^{*}$ & 0.54 & 0.32 & $0.11-0.92$ & 0.04 \\
$\geq 4$ times & - & - & Ref & - & - \\
\hline
\end{tabular}

Note: Other variables were excluded based on their clinical importance, contribution score in the model and $p>0.25$ in bivariate analysis.

$\mathrm{AOR}$, adjusted odds ratio; $\mathrm{Cl}$, confidence interval; $\mathrm{GBV}$, gender-based violence; ref, reference category; SE, standard error.

$*, p<0.05 ; \beta$ for constant $=-2.06 ; R^{2}=0.29$ (Cox \& Snell), 0.39 (Nagelkerke); Model $\chi^{2}(5)=38.81, p<0.001$

of PPD was found to be higher than the worldwide prevalence rates of $12 \%-15 \%,{ }^{25}$ the results are comparable to those from previous studies in the region, such as in South Africa, where PPD ranges between $21 \%$ and $47 \%{ }^{26} \mathrm{~A}$ recent community assessment for APD in a peri-urban area in Eswatini found a prevalence of $22.7 \% .{ }^{15}$ Expectedly, the prevalence of depression can be higher postpartum compared to antepartum because postpartum women have undergone a recent event of childbirth, which is associated with hormonal and psychological changes that predispose them to PPD., ${ }^{3,27}$ Differences in prevalence rates in studies from other regions can be attributed to differences in study designs, measurement of PPD, characteristics of study populations and variations in the contextual risk factors for maternal mental health disorders in different settings. ${ }^{6}$ Worth noting is that in this study, the proportion of mothers who expressed thoughts of harming themselves or their infants was high. Cox and colleagues ${ }^{20}$ stated that participants who answered 'yes' to item 10 (i.e. thought of harming self or the baby) of the EPDS require further psychological evaluation to ensure their safety and that of the baby. In this study, during data collection, those mothers who screened positive and those who expressed suicidal or infanticidal ideation were referred to the NPRH for further psychological management.

We found that mothers who were unemployed were more likely to be depressed consistent with other studies. ${ }^{27,28,29}$ The effects of unemployment on mental health have been long established including the effects of poverty on mental health. ${ }^{30}$ In Eswatini, about $63 \%$ of the population lives below the poverty line, ${ }^{31}$ and most of the women, more especially in rural areas, are housewives, meaning they rely mainly on their spouses for financial support towards buying necessities for raising their children and therefore are prone to suffer from financial strains which may manifest as depressive illness.
Similar to previous studies,,$^{32,33,34}$ in this study, mothers who reported that the pregnancy for the current baby was unplanned had higher odds of being depressed. Unplanned and unwanted pregnancy can lead to lack of acceptance of the child and poor support from the partner which may lead to depression. ${ }^{35}$ In line with previous research, ${ }^{27,36}$ we found that mothers who reported receiving poor social support from their partners had higher odds of being depressed. Shrivastava and colleagues ${ }^{37}$ stated that the most important risk factor for antenatal/postnatal depression is absence of family support especially support from the partner. Eswatini is a patriarchal society, where most of the roles of child-rearing are left to the mother with little involvement of the partner more especially during the first 3 months of the baby's life. In fact, in Swazi culture, new mothers are expected to stay indoors for the first month of the baby's life to protect the baby from 'catching' evil spirits ('kuhabula'). During this time (a period in which she is called an 'umtedlane'), the new mother is often isolated and may even be made to sleep in a separate room as she is not expected to do any house chores in order to promote involution and perineal healing. The primipara (even if she is married) is often allowed to go and stay with her biological mother for a period of about 1-3 months immediately after childbirth so that she can be mentored on how to become a mother. It is during such times that these mothers have little contact and support from their partners, yet practical and emotional support is an important factor during the postpartum period.

Mothers who reported having had fewer than four antenatal care visits had lower odds of screening positive for PPD in our sample. Based on literature, ${ }^{38,39}$ we had expected that women who had more antenatal care (ANC) visits (a sign of good health-seeking behaviour) would have lower odds of being depressed due to the benefits of counselling and educational sessions about transitioning to parenthood offered during ANC, which prepares mothers for postpartum adjustment. The cross-sectional nature of the study limits us to determining temporality and thus impedes our efforts accurately to interpret this finding. For example, it is difficult to infer that women who were depressed during the postpartum period had been depressed since antepartum and therefore were the ones who had higher ANC visits. In fact, that postulation is further disputed by a Ghanaian study ${ }^{40}$ that did not find evidence of an association between APD and antenatal care attendance. In this study, we had asked participants whether they were diagnosed with depression during ANC or not, of which a majority ( $n=71$, $62.3 \%$ ) stated that they were never screened at all for APD, while only three participants $(n=3)$ stated that they were diagnosed with APD (data not shown). The lack of data on APD status on the majority of our sample further impedes the full interpretation of this finding.

The other reason for the unexpected direction of the association between ANC attendance and PPD could be that there are no formal ANC classes about transitioning to parenthood in the public healthcare system in Eswatini, 
yet in literature the women would be expected to benefit from those classes by being empowered on how to develop coping motherhood strategies during the postpartum period. Eswatini does not have a preconception care programme and therefore mothers rely on their past mothering experiences or socialisations by their biological mothers on how to cope with the demands of parenthood. Therefore, we cannot rule out the possibility that the negative association might be due to residual confounding from an unmeasured factor which we did not control for in our model. We say that because in Eswatini, more especially, there is little information shared with the women during ANC about transitioning to parenthood (as might be the case in developed societies). As much as there are usual morning group health education sessions during each ANC visit, those sessions are usually about general $\mathrm{MCH}$ topics and not specific to pregnant women as the audiences comprise all women (including nonpregnant ones) who have come for different $\mathrm{MCH}$ services. Other than that, it is also possible that the unexpected inverse association between ANC attendance and PPD could also be due to lack of statistical power in this study. We suggest that future studies with larger samples explore this association even further.

\section{Strengths and limitations}

To our knowledge, this study is among the first to be published on PPD in Eswatini, which makes our findings important for the $\mathrm{MoH}$ programming as it provides baseline data on maternal mental health in the country. We utilised a high volume facility in the central part of Eswatini and in a region that resembles the country's demographic distribution, which enhances generalisability of our findings within the Manzini region. That said, the nature of the study design cannot be immune from limitations. Firstly, we acknowledge that the limited sample size in this study might have reduced the study's power to detect statistically significant differences between groups. Even though we attempted to demonstrate the robustness of our conservative sample size by fitting a stable regression model on the data, which enhanced the internal validity of our study, the small numbers and resulting large CIs call for cautious interpretation of the findings. The small sample size also limited adjustment of more confounders, which makes our study not to be free from residual confounding.

Secondly, the convenience sampling technique used in this study does not free the study from selection bias.

However, we believe that this did not impact on our findings as we report a very low rate of refusals, of which we would not expect the four participants who declined participation to have been significantly different by a confounding factor from those who remained. We say that because those who refused cited lack of time as a reason and not some other factor that was related to the outcome variable. Thirdly, this was a cross-sectional study and therefore temporality and causality could not be established.

\section{Recommendations}

Despite the weaknesses mentioned previously, we have recommendations to make based on these study findings. The high levels of PPD and suicidal ideation among our sample call for qualitative studies to investigate the psychosocial challenges faced by postpartum women in Eswatini in order to develop psychosocial programmes that will help reduce the incidence of PPD among new mothers. Future studies with larger sample sizes should also investigate the association between antenatal care attendance and postpartum depression. Lastly, the Ministry of Health should consider integrating mental health screening into routine $\mathrm{MCH}$ care in order to detect and treat cases of PPD early. Such screening can also be extended to the antepartum period in order to identify mothers at risk of PPD early and institute prophylactic measures.

\section{Conclusion}

We found a high prevalence of PPD in our sample. Unemployment, unplanned pregnancy and poor social support from partners were found to be the risk factors for PPD. However, attending antenatal care classes fewer times than the WHO-recommended minimum of four visits was found to be protective against PPD. There is a need for longitudinal studies with large samples drawn from the four regions in Eswatini that will examine the mechanisms through which the identified factors influence the development of PPD.

\section{Acknowledgements}

The authors thank the mothers who agreed to participate in this study, as well as the facility administrators for granting permission to conduct this study in their facility.

\section{Competing interests}

The authors declare that they have no financial or personal relationships that may have inappropriately influenced them in writing this article.

\section{Authors' contributions}

L.P.D. conceived the research idea, developed the study protocol, designed the methods, conducted data collection and bivariate data analysis, and wrote the first draft of the manuscript. S.M. provided technical guidance on maternal child health and overall supervision of the study from protocol writing, study implementation and reviewed the manuscript. S.D.D. provided technical guidance on mental health and affective disorders, guided institutional compliance with research ethics, reviewed the study protocol as well as the manuscript. M.C.S. provided methodological and statistical guidance during study conception and protocol writing, supervised data collection, conducted the multivariate statistical analysis and extensively reviewed and revised the manuscript. All authors read and approved the final manuscript. 


\section{Funding information}

This research received no specific grant from any funding agency in the public, commercial, or not-for-profit sectors.

\section{Data availability statement}

The data that support the findings of this study are available from the corresponding author, upon reasonable request.

\section{Disclaimer}

The views, opinions, assumptions or any other information presented in this manuscript are those of the authors and do not necessarily reflect the official policy or position of any affiliated agency of the authors.

\section{References}

1. Stewart $\mathrm{DE}$, Robertson $\mathrm{E}$, Dennis $\mathrm{CL}$, Grace $\mathrm{SL}$, Wallington T. Postpartum depression: Literature review of risk factors and interventions [homepage on the Internet]. Toronto, ON: Toronto Public Health; 2003 [cited 2019 Mar 28] Available from: https://www.who.int/mental_health/prevention/suicide/lit review_postpartum depression.pdf.

2. World Health Organization (WHO). Maternal mental health. Geneva: WHO; n.d Available from: https://www.who.int/mental_health/maternal-child/maternal_ mental_health/en/.

3. American Psychiatric Association. Diagnostic and statistical manual of mental disorders. 5th ed. Arlington: American Psychiatric Association; 2013.

4. Djoda AN, Foumane P, Kamga OJP, Sama DJ, Ngo UME, Mboudou E. Prevalence and risk factors of postpartum depression in Yaounde, Cameroon. Open J Obstet Gynecol. 2015;5(11):608-617. https://doi.org/10.4236/ojog.2015.511086

5. Odinka JI, Nwoke M, Chukwuorji JBC, et al. Post-partum depression, anxiety and marital satisfaction: A perspective from Southeastern Nigeria. S Afr J Psychiat. 2018;24(0),a1109. https://doi.org/10.4102/sajpsychiatry.v24i0.1109

6. Perinatal Mental Health Project. Maternal mental health: Addressing key vulnerabilities. Policy Brief 15 [homepage on the Internet]. 2010 [cited 2019 Mar 28]. Available from: http://www.health.uct.ac.za/usr/health/research/groupings/ mhapp/policy_briefs/MHPB15.pdf.

7. Cox JL. Childbirth as a life event: Sociocultural aspects of postnatal depression. Acta Psychiatr Scand. 1988;344 Suppl:75-83. https://doi.org/10.1111_j.1600-0447.1988. tb09005.x

8. Vigod SN, Villegas L, Dennis CL, Ross LE. Prevalence and risk factors for postpartum depression among women with preterm and low-birth-weight infants: systematic review. BJOG. 2010;117(5):540-550. https://doi. org/10.1111/j.1471-0528.2009.02493.x

9. Fisher J, Cabral de Mello M, Patel V, et al. Prevalence and determinants of common perinatal mental disorders in women in low- and lower-middle-income countries: A systematic review. Bull World Health Organ. 2012;90(2):139-149G. https://doi.org/10.2471/BLT.11.091850

10. Kumwar D, Corey EK, Sharma P, Risal A. Screening for postpartum depression and associated factors among women who deliver at a University Hospital, Nepal. Kathmandu Univ Med J (KUMJ). 2015;13:44-48. https://doi.org/10.3126/kumj. v13i1.13752

11. Eckerdal P, Georgakis MK, Kollia N, Wikstrom A-K, Hogberg U, Skalkidou A Delineating the association between mode of delivery and postpartum depression
symptoms: A longitudinal study. Acta Obstet Gynecol Scand. 2018; 97:301-311. symptoms: A longitudinal study. Acta
https://doi.org/10.1111/aogs.13275

12. Kaya $L$, Çiğdem $Z$. The relationship between mode of delivery and postpartum depression. J Edu Health Promot. 2019;8:5. https://doi.org/10.4103/jehp. jehp_97_18

13. Houston KA, Kaimal AJ, Nakagawa S, Gregorich SE, Yee LM, Kuppermann M Mode of delivery and postpartum depression: The role of patient preferences. Am J Obstet Gynecol. 2015;212(2):229.e1-299.e7. https://doi.org/10.1016/j. ajog.2014.09.002

14. Gold K, Spangenberg K, Wobild P, Schwenke TL. Depression and risk factors for depression among mothers of sick infants in Kumasi, Ghana. Int J Gynaecol Obstet. 2013;130(3):228-231. https://doi.org/10.1016/j.ijgo.2012.09.016.

15. Målqvist M, Clarke K, Matsebula T, Bergman M, Tomlinson M. Screening for antepartum depression through community health outreach in Swaziland. J Community Health. 2016;41:946-942. https://doi.org/10.1007/s10900-016-0175-9
16. Sit D, Luther J, Buysse D, et al. Suicidal ideation in depressed postpartum women: Associations with childhood trauma, sleep disturbance and anxiety. J Psychiatr Res. 2015;66-67:95-104. https://doi.org/10.1016/j.jpsychires.2015. 04.021

17. Ministry of Health. Manzini regional health performance report. Mbabane: Strategic Information Department, Monitoring and Evaluation Unit; 2016.

18. Central Statistical Office (CSO) [Swaziland], Macro International Inc. Swaziland demographic and health survey 2006-07. Mbabane: Government of the Kingdom of Swaziland; 2008.

19. Raosoft I. Raosoft online sample size calculator [homepage on the Internet] Seattle, WA: Raosoft, Inc.; 2004 [cited 2019 Mar 28]. Available from: http://www. raosoft.com/samplesize.html.

20. Cox JL, Holden JM, Sagovsky R. Detection of postnatal depression: Development of the 10-item Edinburgh Postnatal Depression Scale. Br J Psychiatry. 1987;150: 782-786. https://doi.org/10.1192/bjp.150.6.782

21. Tsai AC, Scott JA, Hung KJ, et al. Reliability and validity of instruments for assessing perinatal depression in African settings: Systematic review and meta-analysis. PLoS One. 2013;8(12):e82521. https://doi.org/10.1371/journal.pone.0082521

22. IBM Corp. IBM SPSS Statistics for Windows, version 20.0. Armonk, NY: IBM Corp.; 2011.

23. Field A. Discovering statistics using IBM SPSS Statistics. 5th ed. London: Sage; 2013.

24. Hosmer DWJ, Lemeshow S, Sturdivant RX. Model-building strategies and methods for logistic regression. In: Applied logistic regression. Wiley Series in Probability and Statistics. 3rd ed. Hoboken, NJ: John Wiley \& Sons; 2013. https://doi.org/ $10.1002 / 9781118548387 . c h 4$

25. Stellenberg EL, Abrahams JM. Prevalence of and factors influencing postnata depression in a rural community in South Africa. Afr J Prim Health Care Fam Med. 2015;7(1):a874. https://doi.org/10.4102/phcfm.v7i1.874

26. Heyningen TV, Myer L, Onah M, Tomlinson M, Field S, Honikman S. Antenata depression and adversity in urban South Africa. J Affect Disord. 2016;203:121-129. https://doi.org/10.1016/j.jad.2016.05.052

27. Yusuff ASM, Tang L, Binns CW, Lee AH. Prevalence and risk factors for postnatal depression in Sabah, Malaysia: A cohort study. Women Birth. 2014;28(1):25-29. https://doi.org/10.1016/j.wombi.2014.11.002

28. Shivalli S, Gururaj N. Postnatal depression among rural women in South India: Do socio-demographic, obstetric and pregnancy outcome have a role to play? PLOS One. 2015;10(4):e0122079. https://doi.org/10.1371/journal.pone.0122079

29. Deng AW, Xiong RB, Jiang TT, Luo YP, Chen WZ. Prevalence and risk factors of postpartum depression in a population-based sample of women in Tangxia
Community, Guangzhou. Asian Pac J Trop Med. 2014;7(3):244-249. https://doi. org/10.1016/S1995-7645(14)60030-4.

30. Saurel-Cubizolles M, Romito P, Ancel P-Y, Lelong N. Unemployment and psychological distress one year after childbirth in France. J Epidemiol Community Health. 2000;54(3):185-191. https://doi.org/10.1136/jech.54.3.185

31. Ministry of Health. Annual National Tuberculosis Control Program (NCTP) report, 2016. Mbabane: Ministry of Health; 2017.

32. Brito CN, Alves SV, Ludermir AB, Araújo TV. Postpartum depression among women with unintended pregnancy. Rev Saude Publica. 2015;49:33. https://doi. org/10.1590/S0034-8910.2015049005257

33. Gipson JD, Koenig MA, Hindin MJ. The effects of unintended pregnancy on infant, child, and parental health: A review of the literature. Stud Fam Plann 2008;39(1):18-38. https://doi.org/10.1111/j.1728-4465.2008.00148.x

34. Manikkam L, Burns JK. Antenatal depression and its risk factors: An urban prevalence study in KwaZulu-Natal. S Afr Med J. 2012;102(12):940-944. https:// doi.org/10.7196/samj.6009

35. Cheraghi M, Najafian M, Amoori N, Bazargan A, Cheraghi M, Motaghi M. Risk factors of postpartum depression in Ramhormoz city, Iran. Neuropsychiatria i Neuropsychologia. 2015;10(1):1-4.

36. Al Hinai FI, Al Hinai SS. Prospective study on prevalence and risk factors of postpartum depression in Al-dakhliya governorate in Oman. Oman Med J. 2014;29(3):198-202. https://doi.org/10.5001/omj.2014.49

37. Shrivastava SR, Shrivastava PS, Ramasamy J. Antenatal and postnatal depression: A public health perspective. J Neurosci Rural Pract. 2015;6(1):116-119. https:// doi.org/10.4103/0976-3147.143218

38. Ayele TA, Azale T, Alemu K, Abdissa Z, Mulat H, Fekadu A. Prevalence and associated factors of antenatal depression among women attending antenatal care service at Gondar University Hospital, Northwest Ethiopia. PloS One. 2016;11(5):e0155125-e. https://doi.org/10.1371/journal.pone.0155125

39. Mokhele I, Nattey C, Jinga N, Mongwenyana C, Fox MP, Onoya D. Prevalence and predictors of postpartum depression by HIV status and timing of HIV diagnosis in Gauteng, South Africa. PLoS One. 2019;14(4):e0214849. https://doi.org/10.1371/ journal.pone.0214849

40. Weobong B, Ten Asbroek AHA, Soremekun S, et al. Association of antenata depression with adverse consequences for the mother and newborn in rura Ghana: Findings from the DON population-based cohort study. PLoS One. 2014;9(12):e116333. https://doi.org/10.1371/journal.pone.0116333 